\title{
Pregnenolone stabilizes microtubules and promotes zebrafish embryonic cell movement
}

\author{
Hwei-Jan Hsu ${ }^{1,2}$, Ming-Ren Liang ${ }^{3}$, Chao-Tsen Chen ${ }^{3}$ \& Bon-chu Chung ${ }^{1}$
}

Embryonic cell movement is essential for morphogenesis and the establishment of body shapes ${ }^{1,2}$, but little is known about its mechanism. Here we report that pregnenolone, which is produced from cholesterol by the steroidogenic enzyme Cyp11a1 (cholesterol side-chain cleavage enzyme, $\mathrm{P} 450 \mathrm{scc})^{3}$, functions in promoting cell migration during epiboly. Epiboly is a process in which embryonic cells spread from the animal pole to cover the underlying yolk. During epiboly, cyp11a1 is expressed in an extraembryonic yolk syncytial layer ${ }^{4}$. Reducing cyp11a1 expression in zebrafish using antisense morpholino oligonucleotides did not perturb cell fates, but caused epibolic delay. This epibolic defect was partially rescued by the injection of cypl1a1 RNA or the supplementation of pregnenolone. We show that the epibolic delay is accompanied by a decrease in the level of polymerized microtubules, and that pregnenolone can rescue this microtubule defect. Our results indicate that pregnenolone preserves microtubule abundance and promotes cell movement during epiboly.

Cyp11a1 (also known as P450scc) catalyses the first step of steroid synthesis, the conversion of cholesterol to pregnenolone $\left(\mathrm{P}_{5}\right) . \mathrm{P}_{5}$ is then further converted to $17 \alpha$-hydroxypregnenolone by Cyp 17 or to progesterone $\left(\mathrm{P}_{4}\right)$ by $3 \beta$-hydroxysteroid dehydrogenase (Hsd $3 \mathrm{~b}$ ), for the production of other steroids (Fig. 1a). Steroids regulate salt and sugar homeostasis and sexual characteristics in the body, but the functions of steroids during early embryogenesis are unclear. To study their functions, we characterized the genomic structures of zebrafish cyp 11a1 and $h s d 3 b$ (Fig. 1b), and examined their expression during early embryogenesis by in situ hybridization. After fertilization, zebrafish blastomeres located above the yolk undergo rapid cleavage. At this time, cyp11al and $h s d 3 b$ were expressed in the cytoplasm of these blastomeres (Fig. 1c). The blastomeres then move downward to cover the yolk in a process called epiboly. During epiboly, cyp11a1 and $h s d 3 b$ were downregulated from blastomeres, and a new expression domain appeared at the junction between blastomeres and yolk. This expression domain, called the yolk syncytial layer (YSL), is an extra-embryonic layer composed of yolk syncytial nuclei that move ahead of blastomeres during epiboly.

To test the function of cyp11a1 during early embryogenesis, we injected antisense morpholino (mo) oligonucleotides into zebrafish embryos at the one-cell stage to block its expression (Supplementary Fig. S1). At 100\% epiboly, when wild-type blastomeres completely covered the yolk, embryos injected with cyp11a1-mo1 could not cover the yolk, leaving an unclosed yolk plug (Fig. 2). Control embryos injected with morpholino oligonucleotides against a random sequence, an unrelated sequence from $d l x 3, h s d 3 b$ or $c y p 17$, or a 5-base mismatched cyp11a1-mo1 were all normal (Supplementary Table S1). The epibolic delay started before gastrulation, when wild-type blastomeres cover $50 \%$ of the yolk (50\% epiboly). At this time, blastomeres of the cyp11a1-mo1 morphants covered only about
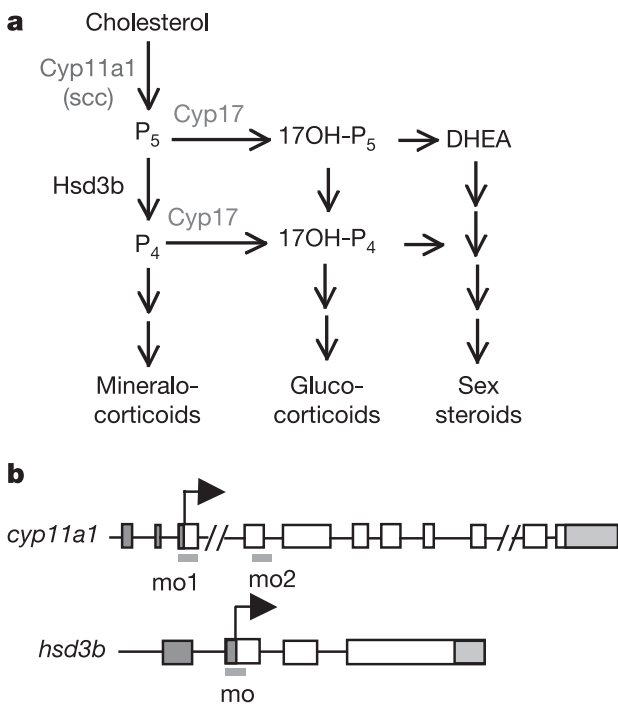

c
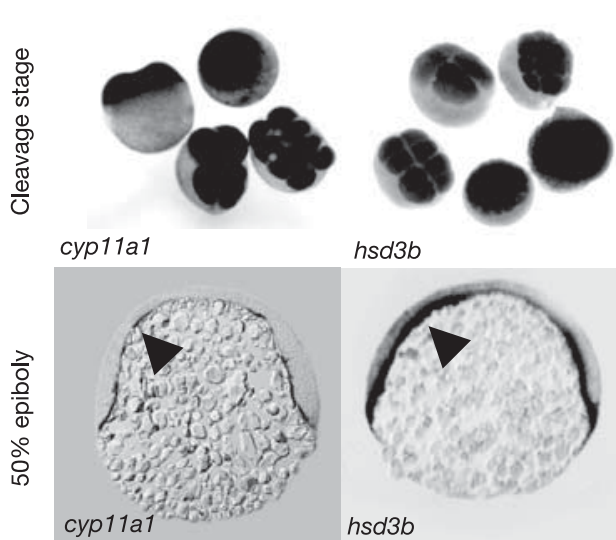

Figure 1 | Two steroidogenic genes, cyp11a1 and hsd3b, are expressed in blastomeres and the yolk syncytial layer during early zebrafish embryogenesis. a, Steroidogenic pathways. Cyp11al catalyses the conversion of cholesterol to pregnenolone $\left(\mathrm{P}_{5}\right)$, which is then converted to $17 \alpha$-hydroxypregnenolone $\left(17 \mathrm{OH}-\mathrm{P}_{5}\right)$ and progesterone $\left(\mathrm{P}_{4}\right)$ by Cyp17 and Hsd3b, respectively. DHEA, dehydroepiandrosterone. b, Genomic structures of zebrafish cyp11al and $h s d 3 b$. Shaded and open boxes indicate coding and non-coding regions of exons, respectively. Arrows indicate transcriptional start sites; lines below the boxes indicate the locations of morpholino oligonucleotides (mo). c, Detection of cyp11a1 and $h s d 3 b$ expression by whole-mount in situ hybridization at the cleavage and $50 \%$ epiboly stages. Arrowheads indicate yolk syncytial layer. 
$44 \%$ of the yolk (data not shown). Thus, cyp11a1-mo1 morphants show an early epibolic defect.

When we injected a fluorescein-conjugated control morpholino oligonucleotide into embryos at the one-cell stage, the fluorescent signal was distributed throughout the entire embryo at $30 \%$ epiboly. When we injected the same morpholino into the yolk at the 1,000 (1k)-cell stage, the fluorescent signal was restricted to only the YSL (Supplementary Fig. S2a). Thus, injecting oligonucleotides into the yolk at the 1k-cell stage affects only YSL function. At 100\% epiboly, embryos injected with cyp11al-mol at the 1k-cell stage failed to close their yolk plug, although the extent of epibolic delay was less severe than that for embryos injected at the one-cell stage (Supplementary Fig. S2b). This result indicates that cyp11a1 expressed in the YSL participates in the epibolic process.

The YSL is known to be involved in mesendoderm induction ${ }^{5}$ and nutrient production ${ }^{6,7}$. To test whether cyp11a1 in the YSL is involved in germ layer induction, we examined cell fates in cyp11a1-mo1 morphants using mesodermal ( $t b x 6$ and $s h h)$, mesendodermal $(g s c)$ and endodermal (sox17) markers. The cell fates of mesoderm and endoderm did not change upon reduction of cyp11a1 expression by cyp11a1-mo1 (Supplementary Fig. S3). Although cell fates of the cyp11a1-mo1 morphants appeared normal, the length of their anterior-posterior axis was reduced (Supplementary Fig. S3d) and their somites were wider (data not shown). This indicates a problem with the convergent extension movement that builds up body axis by narrowing and lengthening dorsal tissues. Not all types of cell movements were affected, as involution movement seemed normal (Supplementary Fig. S4).

To confirm the function of cyp11a1, we used a different morpholino oligonucleotide, cyp11a1-mo2, which targets the junction between exon 4 and intron 4 of cypl1a1, and affects zygotic cyp11a1 RNA splicing and epibolic movement (Fig. 1b and Supplementary Fig. S5). This morpholino also caused epibolic delay. The epibolic delay caused by each of cyp11a1-mo1 and cyp11a1-mo2 was partially rescued when embryos were co-injected with cyp11a1 mRNA (Supplementary Figs S2c and S5d). This result shows that cyp11a1 contributes to epibolic movement.

Cyp11a1 encodes a steroidogenic enzyme (Fig. 1a), and we predicted that reducing cyp11a1 expression would result in a decrease in steroid products. Indeed, the level of the Cyp1la1 product pregnenolone from cyp11a1-mol morphants $10 \mathrm{~h}$ after fertilization was about half of that in wild-type embryos (Supplementary Fig. S1b), and incubation of cyp11a1-mo1 morphants with pregnenolone from the early cleavage stage partially rescued the epibolic defect (Fig. 3a).

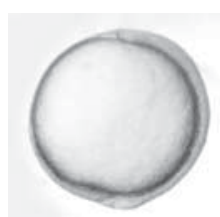

WT

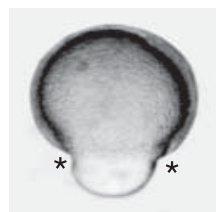

cyp11a1-mo1

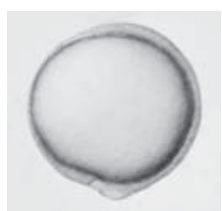

hsd3b-mo

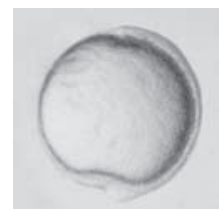

cyp11a1 5-mis

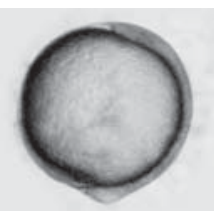

cyp17-mo

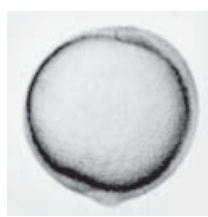

Random mo
Figure 2 | Reduced cyp11a1 expression results in epibolic delay. a, Phenotypic examination of embryos under a dissecting microscope. Wild-type (WT) embryos and embryos injected with control, cyp11a1-mo1, cyp11a1 5-mis (5-base mismatched cyp11a1-mo1), hsd3b or cyp17

morpholino oligonucleotides (mo) at the one-cell stage reach $100 \%$ epiboly. Embryos injected with cyp11a1-mo1 cannot close the yolk plug.
This indicates that pregnenolone and/or its metabolite is involved in zebrafish epiboly.

To determine whether pregnenolone is metabolized further, we incubated embryos with radioactive pregnenolone and checked the formation of metabolites by thin-layer chromatography. We found that some pregnenolone could be metabolized into progesterone, but that very little $17 \alpha$-hydroxypregnenolone was produced, even when Hsd3b activity was knocked down (Supplementary Fig. S6). When tested, progesterone could not rescue the epibolic defect-instead, it worsened the severity of the growth delay (data not shown). As a concentration of only $0.1 \mu \mathrm{M}$ pregnenolone was enough to rescue the epibolic defect (Fig. 3a), and this concentration is lower than the $K_{\mathrm{m}}$ for either Cyp17 (5 $\mu \mathrm{M}$; ref. 8) or Hsd3b $(0.2-1.6 \mu \mathrm{M}$; ref. 9), it is likely that pregnenolone, rather than its metabolite, rescues the epibolic delay.

Zebrafish epiboly is driven by yolk microtubule arrays, which extend from yolk syncytial nuclei along the animal-vegetal axis of the yolk ${ }^{10-12}$. To investigate whether the epibolic function of cyp11a1 is related to microtubules, we visualized microtubules by staining them with an antibody against $\alpha$-tubulin. We observed no significant difference between wild-type and cyp11a1-mol morphants during epiboly (data not shown). We therefore tested the stability of microtubules by treating embryos at $50 \%$ epiboly with the microtubule-disrupting agent nocodazole. While almost completely disrupted by $10 \mu \mathrm{g} \mathrm{ml}^{-1}$ nocodazole ${ }^{11}$ (data not shown), yolk microtubules remained intact with treatment of $1 \mu \mathrm{g} \mathrm{ml}^{-1}$ nocodazole, but the yolk microtubules of cyp11a1-mol morphants were thinner than those of wild-type embryos (Fig. 3b). This result shows that yolk microtubules of cyp11a1 morphants are more sensitive to nocodazole treatment, indicating that they are less stable ${ }^{13,14}$. When cyp11a1-mo1 morphants were incubated with pregnenolone, yolk microtubule structure was restored (Fig. 3b). Therefore, pregnenolone can stabilize yolk microtubules in vivo.

In addition to the imaging study described above, we examined microtubule content using a biochemical method. Protein extracts from wild-type embryos and cyp11a1-mol morphants at 50\% epiboly were centrifuged at $100,000 \mathrm{~g}$ to separate polymerized tubulin (pellet) from free tubulin (supernatant). When embryos were treated with the tubulin-polymerizing drug taxol, the amount of polymerized tubulin in the pellet was increased (Fig. 3c). In cyp11a1-mo1 morphants, the amount of polymerized tubulin in the pellet was much less than for wild type, indicating a lower abundance of polymerized microtubules. When the extract from cyp11a1-mol morphants was incubated with pregnenolone, the amount of polymerized tubulin in the pellet increased in a dosedependent manner (Fig. 3d). This indicates that pregnenolone can increase the amount of polymerized tubulin in vitro.

To visualize the interaction between pregnenolone and microtubules, we conjugated the fluorescent dye fluorescein to pregnenolone (Supplementary Fig. S7). The resulting $\mathrm{F}-\mathrm{P}_{5}$ also increased the abundance of polymerized tubulin in cyp11a1-mol morphant extracts (Supplementary Fig. S8), indicating the fluorescein moiety does not interfere with pregnenolone activity. When incubated with cyp11a1-mol morphant extract for $30 \mathrm{~min}, \mathrm{~F}-\mathrm{P}_{5}$ co-localized with polymerized $\alpha$-tubulin (Fig. 4). When fluorescein alone was incubated with morphant extract, it did not co-localize with $\alpha$-tubulin and microtubules were not detected. This indicates that pregnenolone binds to microtubules, or to something that associates with microtubules in cell extracts. This binding results in increased microtubule content in vitro.

Microtubule dynamics provide an important force for cell movement and neurite outgrowth ${ }^{15,16}$. However, the regulators of microtubules are largely unknown, except for a few identified proteins such as CLASPS, MAP2c, MACF7 and Op18, which stabilize microtubules in various cell types ${ }^{13,17,18}$. Here we show that pregnenolone is a regulator of yolk microtubules_it maintains the abundance of yolk microtubules and promotes epibolic movement. Pregnenolone 
a
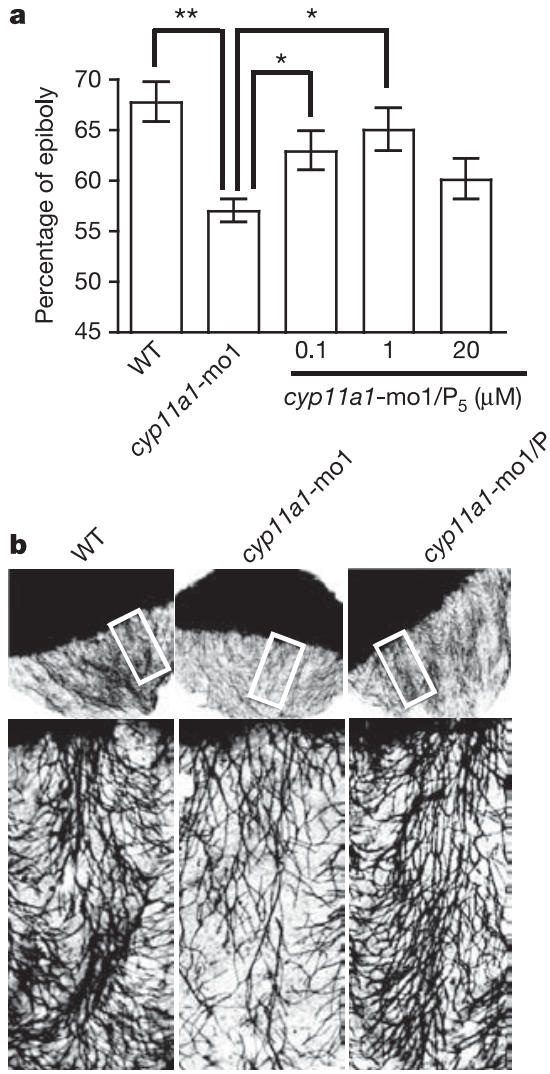

Figure 3 Pregnenolone rescues epiboly defect and increases microtubule content. a, The effect of different concentrations of pregnenolone on the percentage of epiboly. Error bars show s.d. ${ }^{\star} P<0.05 ;{ }^{\star \star} P<0.01$.

b, Microtubule structure of nocodazole-treated wild-type embryos (16 out of 18 embryos showed normal microtubule structure, $n=16 / 18$ ), cyp11a1-mol-injected embryos $(n=19 / 23)$ or cyp11a1-mol morphants incubated with $20 \mu \mathrm{M} \mathrm{P}_{5}(n=19 / 19)$ at $50 \%$ epiboly. Enlarged images show

promotes microtubule assembly through binding to microtubuleassociated protein 2 from rat brain ${ }^{19}$. This provides insight into the mechanism of cell movement controlled by pregnenolone, not only during zebrafish embryogenesis but probably also for neurite outgrowth and other types of cell movement.
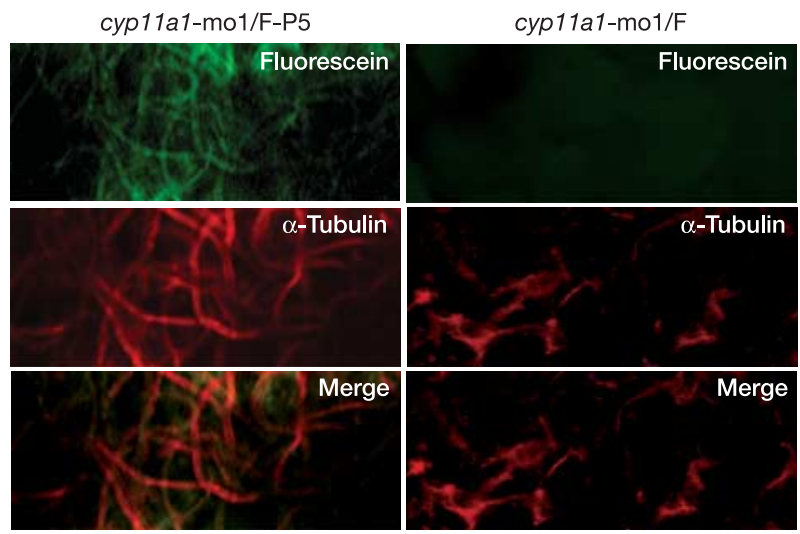

Figure 4 | Fluorescein-conjugated pregnenolone co-localizes with microtubules. Detection of fluorescein and $\alpha$-tubulin in the microtubule pellet after incubation of cyp11a1-mo1-treated embryo extracts with $20 \mu \mathrm{M}$ taxol and fluorescein-conjugated pregnenolone $\left(\mathrm{F}-\mathrm{P}_{5}\right)$ for $30 \mathrm{~min}$ followed by centrifugation. When incubated with taxol and fluorescein alone (F), no microtubule signal was detected (except for some nonspecific tubulin staining of cell debris).

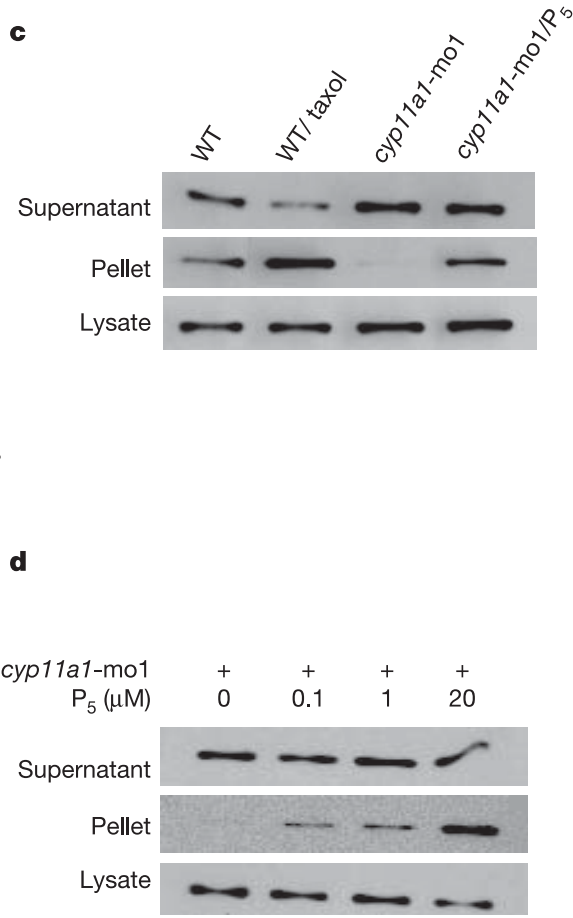

higher magnifications of the areas in the rectangles. c, d, Microtubule content assay. Embryo extracts from wild-type (WT) or cyp11a1-mo1injected embryos at $50 \%$ epiboly were incubated with or without $20 \mu \mathrm{M}$ taxol or different concentrations of $\mathrm{P}_{5}$, centrifuged to separate free tubulin in the supernatant from polymerized microtubules in the pellet, then probed with an $\alpha$-tubulin antibody. mo, morpholino oligonucleotide.

Here we describe a new function for cyp11al expressed in the zebrafish YSL. The YSL is known to have a role in morphogenesis during gastrulation ${ }^{20}$, but how it participates in morphogenetic cell movement was not clear. We now show that the YSL gene cyp11a1 is important for the production of pregnenolone, which preserves the abundance of microtubules, thus promoting cell movement.

The production of steroids from extra-embryonic tissues is not unique to zebrafish. In mouse, steroids are secreted from extraembryonic giant trophoblast cells during early pregnancy ${ }^{21}$, but the function of steroids in mammalian embryogenesis cannot be easily investigated $^{22,23}$. The Drosophila steroid ecdysone is activated in an extra-embryonic tissue and is involved in morphogenetic cell movements for building up the body plan ${ }^{24}$, but its mechanism of action is not clear. Using zebrafish, we have shown that the steroid pregnenolone, produced from an extra-embryonic tissue, participates in early morphogenesis by acting on microtubules. Steroids usually act through a genomic mechanism, whereby steroids bind to steroid receptors and control the expression of target genes ${ }^{25}$. Other non-genomic actions have been proposed for steroids ${ }^{26}$. We now show that pregnenolone promotes cell movements by directly stabilizing microtubules, probably through a non-genomic mechanism.

\section{METHODS}

Microinjection and reagent treatment. Morpholino oligonucleotides (Gene Tools) were diluted to working concentrations of $0.5-3 \mu \mathrm{g} \mu \mathrm{l}^{-1}$ in Danieau solution $\left(58 \mathrm{mM} \mathrm{NaCl}, 0.7 \mathrm{mM} \mathrm{KCl}, 0.4 \mathrm{mM} \mathrm{MgSO}_{4}, 0.6 \mathrm{mM} \mathrm{Ca}\left(\mathrm{NO}_{3}\right)_{2}, 5 \mathrm{mM}\right.$ HEPES, pH 7.6) and injected into the yolk of embryos at the one-cell or $1 \mathrm{k}$-cell stage at a concentration of $9 \mathrm{ng}$ per embryo, unless noted otherwise. For RNA 
rescue, $9 \mathrm{ng}$ cyp11al-mol and $200 \mathrm{pg}$ of cyp11al RNA or $\beta$-gal RNA were coinjected into embryos at the one-cell stage. Embryos were incubated with pregnenolone $(0.1,1$ or $20 \mu \mathrm{M})$ or progesterone $(1$ or $20 \mu \mathrm{M})$ after dechorionation and before the 16-cell stage until the late epiboly stage. For nocodazole treatment, $1 \mu \mathrm{g} \mathrm{ml}^{-1}$ nocodazole was added to the embryo medium at $50 \%$ epiboly for $20 \mathrm{~min}$ at $28.5^{\circ} \mathrm{C}$.

Whole-mount in situ hybridization and immunostaining. Whole-mount in situ hybridization was performed using digoxigenin-labelled antisense RNA probes and alkaline phosphatase-conjugated secondary antibodies, as described previously $\mathrm{y}^{27}$. Photographs were taken using an Olympus BX50 microscope. Microtubule staining with an anti- $\alpha$-tubulin antibody was performed according to a previously described procedure ${ }^{28}$. Fluorescent images were captured by Zeiss LSM 510 confocal microscope. Tubulin images were processed by the $3 \mathrm{D}$ visualization and deconvolution LSM510 basis software (v3.0, Zeiss).

Measurement of the extent of epiboly. Embryos were hybridized with an $n t l$ probe to mark the marginal blastomeres, and photos were taken using a Leica MZFLIII microscope with a CCD camera (Diagnostic Instrument Inc). The length from animal pole to vegetal pole and the height of the blastoderm for each embryo were measured by SPOT program (v.4-0-8, Diagnostic Instrument Inc) (Supplementary Fig. S9). The distance of the blastoderm margin divided by the animal-vegetal length is regarded as the percentage of epiboly.

Synthesis of fluorescein-conjugated pregnenolone $\left(\mathrm{F}-\mathrm{P}_{5}\right) . \mathrm{F}-\mathrm{P}_{5}$ consists of fluorescein and pregnenolone bridged by a six-carbon linker via ethereal and thioureido functionalities (see Supplementary Information). It was prepared in six steps with $14 \%$ overall yield, starting from commercially available pregnenolone.

Microtubule abundance assay. This procedure was according to the instructions provided with the microtubules/tubulin in vivo assay kit (Cytoskeleton). Twenty embryos injected with or without $9 \mathrm{ng}$ cyp 11a1-mol were placed in $200 \mu \mathrm{l}$ lysis buffer plus $1 \mathrm{mM}$ GTP, $10 \mathrm{mM}$ ATP and $20 \mu \mathrm{l}$ proteinase inhibitor cocktail, homogenized, then incubated with taxol $(20 \mu \mathrm{M})$, pregnenolone $(0.1,1,20 \mu \mathrm{M})$, fluorescein or fluorescein-conjugated pregnenolone at $30^{\circ} \mathrm{C}$ for $30 \mathrm{~min}$. A $180-\mu \mathrm{l}$ aliquot was transferred into a pre-warmed tube and centrifuged at $100,000 \mathrm{~g}, 30^{\circ} \mathrm{C}$ for $30 \mathrm{~min}$. After centrifugation, pellets were dissolved in $180 \mu \mathrm{l}$ cold $200 \mu \mathrm{M} \mathrm{CaCl}_{2}$. For western blot analysis, $2.5 \mu \mathrm{l}$ each of total lysate and supernatant samples, and $15 \mu \mathrm{l}$ of pellet samples were loaded onto the gels. For the imaging study, microtubule pellets were removed from centrifuged tubes and stained with $\alpha$-tubulin antibody, followed by spotting onto a glass slide for fluorescence detection.

\section{Received 9 September; accepted 25 October 2005.}

1. Locascio, A. \& Nieto, M. A. Cell movements during vertebrate development: integrated tissue behaviour versus individual cell migration. Curr. Opin. Genet. Dev. 11, 464-469 (2001)

2. Myers, D. C., Sepich, D. S. \& Solnica-Krezel, L. Convergence and extension in vertebrate gastrulae: cell movements according to or in search of identity? Trends Genet. 18, 447-455 (2002).

3. Miller, W. L. Molecular biology of steroid hormone synthesis. Endocr. Rev. 9, 295-318 (1988)

4. Hsu, H. J., Hsiao, P., Kuo, M. W. \& Chung, B. C. Expression of zebrafish cyp11a1 as a maternal transcript and in yolk syncytial layer. Gene Expr. Patterns 2, 219-222 (2002)

5. Chen, S. \& Kimelman, D. The role of the yolk syncytial layer in germ layer patterning in zebrafish. Development 127, 4681-4689 (2000).

6. Babin, P. J. et al. Both apolipoprotein E and A-I genes are present in a nonmammalian vertebrate and are highly expressed during embryonic development. Proc. Natl. Acad. Sci. USA 94, 8622-8627 (1997).

7. Sharma, M. K. et al. Sequence, linkage mapping and early developmental expression of the intestinal-type fatty acid-binding protein gene (fabp2) from zebrafish (Danio rerio). Comp. Biochem. Physiol. B Biochem. Mol. Biol. 138, 391-398 (2004).

8. Grigoryev, D. N. et al. Cytochrome P450c17-expressing Escherichia coli as a first-step screening system for $17 \alpha$-hydroxylase- $C_{17,20}$-lyase inhibitors. Anal. Biochem. 267, 319 (1999).
9. Rheaume, E. et al. Structure and expression of a new complementary DNA encoding the almost exclusive 3 beta-hydroxysteroid dehydrogenase/delta 5-delta 4-isomerase in human adrenals and gonads. Mol. Endocrinol. 5, 1147-1157 (1991)

10. Jesuthasan, S. \& Stahle, U. Dynamic microtubules and specification of the zebrafish embryonic axis. Curr. Biol. 7, 31-42 (1997).

11. Solnica-Krezel, L. \& Driever, W. Microtubule arrays of the zebrafish yolk cell: organization and function during epiboly. Development 120, 2443-2455 (1994).

12. Strahle, U. \& Jesuthasan, S. Ultraviolet irradiation impairs epiboly in zebrafish embryos: evidence for a microtubule-dependent mechanism of epiboly. Development 119, 909-919 (1993).

13. Akhmanova, A. et al. Clasps are CLIP-115 and -170 associating proteins involved in the regional regulation of microtubule dynamics in motile fibroblasts. Cell 104, 923-935 (2001).

14. Liu, L., Tommasi, S., Lee, D. H., Dammann, R. \& Pfeifer, G. P. Control of microtubule stability by the RASSF1A tumour suppressor. Oncogene 22, 8125-8136 (2003)

15. Dent, E. W. \& Gertler, F. B. Cytoskeletal dynamics and transport in growth cone motility and axon guidance. Neuron 40, 209-227 (2003).

16. Watanabe, T., Noritake, J. \& Kaibuchi, K. Regulation of microtubules in cell migration. Trends Cell Biol. 15, 76-83 (2005).

17. Morris, N. R. Nuclear positioning: the means is at the ends. Curr. Opin. Cell Biol. 15, 54-59 (2003).

18. Wittmann, T., Hyman, A. \& Desai, A. The spindle: a dynamic assembly of microtubules and motors. Nature Cell Biol. 3, E28-E34 (2001).

19. Murakami, K., Fellous, A., Baulieu, E. E. \& Robel, P. Pregnenolone binds to microtubule-associated protein 2 and stimulates microtubule assembly. Proc. Natl Acad. Sci. USA 97, 3579-3584 (2000).

20. D'Amico, L. A. \& Cooper, M. S. Morphogenetic domains in the yolk syncytial layer of axiating zebrafish embryos. Dev. Dyn. 222, 611-624 (2001).

21. Arensburg, J., Payne, A. H. \& Orly, J. Expression of steroidogenic genes in maternal and extraembryonic cells during early pregnancy in mice. Endocrinology 140, 5220-5232 (1999).

22. Hu, M. C. et al. Steroid deficiency syndromes in mice with targeted disruption of Cyp11a1. Mol. Endocrinol. 16, 1943-1950 (2002).

23. Venihaki, M., Carrigan, A., Dikkes, P. \& Majzoub, J. A. Circadian rise in maternal glucocorticoid prevents pulmonary dysplasia in fetal mice with adrenal insufficiency. Proc. Natl. Acad. Sci. USA 97, 7336-7341 (2000).

24. Kozlova, T. \& Thummel, C. S. Essential roles for ecdysone signaling during Drosophila mid-embryonic development. Science 301, 1911-1914 (2003).

25. Cato, A. C., Nestl, A. \& Mink, S. Rapid actions of steroid receptors in cellular signaling pathways. Sci. STKE 2002, RE9 (2002).

26. Schmidt, B. M. et al. Rapid, nongenomic steroid actions: A new age? Front. Neuroendocrinol. 21, 57-94 (2000).

27. Chiang, E. F. et al. Two sox9 genes on duplicated zebrafish chromosomes: expression of similar transcription activators in distinct sites. Dev. Biol. 231, 149-163 (2001).

28. Gard, D. L. Organization, nucleation, and acetylation of microtubules in Xenopus laevis oocytes: a study by confocal immunofluorescence microscopy. Dev. Biol. 143, 346-362 (1991).

Supplementary Information is linked to the online version of the paper at www.nature.com/nature.

Acknowledgements We would like to thank V. Korzh and C.-H. Hu for the plasmids for in situ hybridization, and K. Deen and M. Wyatt for editing the manuscript. This work was supported by grants from the National Science Council and Academia Sinica, Taiwan.

Author Contributions H.-J.H. performed all the experiments; M.-R.L. synthesized $\mathrm{F}_{-} \mathrm{P}_{5}$; C.-T.C. devised and supervised the $\mathrm{F}-\mathrm{P}_{5}$ synthesis scheme; B.-c.C. oversaw the execution of the entire project.

Author Information Reprints and permissions information is available at npg.nature.com/reprintsandpermissions. The authors declare no competing financial interests. Correspondence and requests for materials should be addressed to B.-c.C. (mbchung@sinica.edu.tw). 